\title{
The potential application of cereal straw as a bio-filler for elastomer composites
}

\author{
Marcin Masłowski ${ }^{1}$ D J Justyna Miedzianowska ${ }^{1} \cdot$ Krzysztof Strzelec $^{1}$
}

Received: 22 January 2019 / Revised: 2 April 2019 / Accepted: 17 June 2019 / Published online: 20 June 2019 (c) The Author(s) 2019

\begin{abstract}
The purpose of this research article is to present the application possibilities of cereal straw as a functional bio-filler of elastomeric materials. The work defined the precise selection of polymeric matrixes as a base for the produced biocomposites. Applied rubbers, ethylene propylene, methylvinylsilicone, butadiene-styrene and butadiene-acrylonitrile, are materials that differ in both chemical structure and methods of cross-linking. Due to the differences in material characteristics, the composites presented specific properties, which have been confirmed by a number of research methods that determine rheometric, mechanical, dynamical-mechanical properties, damping, cross-linking density and susceptibility to simulated thermooxidative aging processes. Application of straw as a natural bio-fillers for different elastomers increased cross-linking densities of the composites and reduced the curing time of the mixtures. Straw vulcanizates, regardless of the polymer type and simultaneously with increasing filling degree, showed a significant increase in tear strength and hardness. Moreover, these materials showed high values of damping coefficients and satisfactory strength properties, depending on the mechanical characteristics of the rubbers used. Furthermore, biocomposites produced on the basis of ethylene propylene and silicone rubber have proven their high resistance to simulated aging processes. The use of ground straw as a never previously used filler of elastomer composites proves that it is a highly valuable additive both in economic and material aspects. Its application in elastomer technology may contribute to the management of surplus agricultural wastes while being used in materials exhibiting useful properties.
\end{abstract}

Keywords Cereal straw $\cdot$ Elastomer composites $\cdot$ Rubbers $\cdot$ Bio-filler $\cdot$ Agriculture waste

Marcin Masłowski

marcin.maslowski@p.lodz.pl

1 Institute of Polymer and Dye Technology, Lodz University of Technology, Stefanowskiego

12/16, 90-924 Lodz, Poland 


\section{Introduction}

Polymeric biocomposites filled with natural fibers (NFC) become an alternative to composites reinforced with synthetic fibers. The increased development of research on NFCs materials is primarily due to the benefits of their potential applications in many industrial areas around the world. In addition, composite materials based on bio-additives provide an alternative to the constantly depleting, non-renewable conventional sources [1-4]. Good electrical resistance, improved thermal and acoustic insulation properties, high resistance to cracking and above all, very good mechanical properties are undoubtedly one of the most important characteristics and the predominance of natural fiber polymer composites. Very important are also the issues of the possibility of reducing the production costs of these materials while maintaining correspondingly attractive functional properties [2, 5-7]. The construction, packaging, sports, automotive, recreational and aerospace industries are just the beginning of the main applications of biocomposites, in the form of, among others, window and door frames, finishing panels, pipes, laminated boards, upholstery and car accessories, packaging, garden furniture, railway sleepers and many other [8-10].

Natural fibers are generally classified into two main types: animal-based fibers with cocoon silk, chicken feather, wool or spider silks as representatives and plant-based natural fibers including, straw, jute, hemp, sisal, kenaf, coir, flax, bamboo, pineapple and banana [11]. Natural plant-based fibers are lignocellulosic materials consisting of cellulose, hemicellulose and lignin, demonstrating solid advantages such as availability, high growth rate, renewability, low cost, low weight and high specific strength and stiffness. In spite of the above, natural fibers also show drawbacks such as high porosity, high moisture content, difficulty in extracting fine and continuous fibers as well as thermal degradation during the production process, which hinder some of their applications [12].

Ecological aspects of biocomposites are clarified and exactly stated; however, producers of biocomposite materials do not fully exploit their potential. A certain alternative in overcoming problems resulting from the properties of fibers is the matching and choice of polymers in order to obtain a proper bond between natural fibers and the matrix influencing its further characteristics like: providing a barrier against adverse environments, protecting the surface of the fibers from mechanical abrasion and transfer load to fibers [13]. The most important factor affecting the selection of polymers is the possibility of processing them at temperatures below $200{ }^{\circ} \mathrm{C}$, which results from the thermal stability of natural fibers. Therefore, it is common to use thermoplastics such as polyethylene (PE), polypropylene (PP), polyvinyl chloride (PCV) and polystyrene (PS), as well as thermosetting resins [14-16]. The presented limitations create possibilities for using elastomeric materials, for which the standard processing temperature is ca. $150-180{ }^{\circ} \mathrm{C}$. Elastomeric mixtures after the cross-linking (vulcanization) process, so-called rubbers are extremely valuable materials with a huge application spectrum, depending on the type of polymer. So far, apart from natural rubber, there are no literature reports on the widespread use of these plastics as a base for the 
production of biocomposites with the addition of natural fibers. The appropriate material combination of both the elastomeric medium and the bio-filler can provide functional properties of such composites resulting from the specific characteristics of both polymeric matrices and the potential of natural fibers.

Undoubtedly, cereal straw is found to be very interesting and promising functional filler for natural rubber composites which has been confirmed in several publications [17-19]. Straw is a lignocellulosic biomass material, a by-product of agricultural crops production containing mainly: $35-50 \%$ of cellulose, $15-30 \%$ of hemicellulose and $20-30 \%$ of lignin. Currently, its final uses on the farm are primarily animal bedding and feeding. However, the vast majority of straw produced remains in the field to spread or be burnt, which is not without reason causing environmental concerns in many countries. Considering the commercial possibilities of straw applications, it is worth mentioning the production of electricity and heat through combustion and gasification, ethanol production through microbial fermentation, methane production through anaerobic digestion, fungal growth, construction of buildings and production of composite and paper products and control of soil erosion [20, 21]. Generally, although cereal straw is a plentiful source of biomass, its actual use is only a small percentage of its potential; hence, the search for new solutions, such as the addition of elastomer composites, is particularly desirable.

The aim of this research article was to obtain elastomeric compositions with the addition of ground cereal straw as a lignocellulosic filler and an in-depth characterization of their functional properties. As elastomeric matrices, four types of rubbers (ethylene propylene, silicone, butadiene-styrene and butadiene-acrylonitrile) were used, differing in chemical structure and polarity, and thus in the use of cross-linking agents. Due to the various elastomer applied, the biocomposites obtained will exhibit characteristics specific to a given material group. This creates the possibility of designing composites with strictly defined properties, which in the future can be tailored to the requirements of potential consumers from multiple industries. The straw came from several species of cereals (rye, wheat, oat, triticale, barley and corn) collected from the same territorial regime milled together to obtain a bio-filler in the form of a fine powder. The work is based on the innovative use of straw as lignocellulosic filler acting as a functional additive to elastomer matrices not described previously in the subject literature. The full material characterization of both elastomer mixtures and finished products (vulcanizates) was examined, including rheometric, damping and mechanical properties, cross-linking density, tear strength, dynamic mechanical analysis (Payne effect), thermo-oxidative aging resistance and hardness.

\section{Experimental}

\section{Materials}

As the elastomer matrixes were used: 
- Ethylene propylene polymer (EPM) (Dutral CO 054) with propylene content of $41 \%$, purchased from Versalis S.p.A. (Italy).

- Methylvinylsilicone rubber (MVQ) (POLIMER MV 0.07), containing $0.07 \%$ of vinyl groups, purchased from Chemical Plant "Silikony Polskie" (Poland).

- Butadiene-styrene rubber (SBR) (KER 1500) containing 22-25\% styrene, purchased from Synthos S.A. (Poland).

- Acrylonitrile-butadiene rubber (NBR) (Europrene N2845 GRN) with an acrylonitrile content of $28 \%$, purchased from Torimex-Chemicals Ltd. Sp. z o.o (Poland).

The straw filler was collected from local farms, coming from various types of cereals: wheat, rye, oat, triticale and barley. Straw stalks were cut into $10 \mathrm{~mm}$ pieces and grinding using a ball mill for $0.5 \mathrm{~h}$ (SPEX SamplePrep 8000D Mixer/Mill, Metuchen, UK).

The composition of the elastomeric mixtures is shown in Table 1. NBR and SBR rubbers were vulcanized with sulfur system: sulfur $\left(\mathrm{S}_{8}\right)$ (Siarkopol, Poland), microsized zinc oxide ( $\mathrm{ZnO}$ ) (Huta Bedzin, Poland), 2-mercaptobenzothiazole (MBT) (Sigma-Aldrich, Germany) and stearic acid (SA, Avantor Performance Materials, Poland) (Table 1a). While the EPM and MVQ elastomers were cross-linked with dicumyl peroxide (DCP) (Sigma-Aldrich, Germany) (Table 1b).

\section{Methods}

Rubber mixtures were prepared in two steps using measuring mixer and laboratory two-roll mill. First, rubber was mixed for 4 min in a measuring mixer (Brabender N50, Duisburg, Germany). By pre-mixing, pure elastomer decreased its viscosity by friction generated heat in order to ease the incorporation of the rest of the ingredients. Then, mixing was continued with the addition of straw for another 4 min (for filled composites). The speed of rotors was $40 \mathrm{rpm}$, and an average temperature was $40-50{ }^{\circ} \mathrm{C}$. In the next step, the two-roll mill was used to mix filled and unfilled rubber blends with the curing systems and obtain elastomer sheets.

Table 1 The composition of the elastomeric mixtures cured with (a) sulfuric crosslinking system (b) peroxide cross-linking system

\begin{tabular}{lllllll}
\hline Component & Rubber: SBR, NBR & Filler: cereal straw & ZnO & MBT & Sulfur & Stearic acid
\end{tabular}

(a) Sulfuric cross-linking system

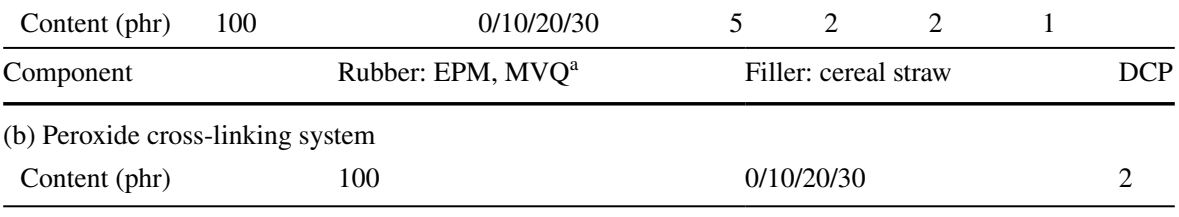

phr parts per hundred parts of rubber

${ }^{a}$ All MVQ composites were filled with $15 \mathrm{phr}$ of silica [Aerosil A380, hydrophilic fumed silica with a specific surface area of $380 \mathrm{~m}^{2} / \mathrm{g}$ (Evonik, Germany)] 
The cure properties of rubber mixtures were measured at $160{ }^{\circ} \mathrm{C}$ by a rheometer with the oscillating disk (Alpha Technologies, USA) in accordance with ISO 3417. The cure properties including scorch time $\left(t_{\mathrm{s} 2}\right)$, optimum cure time $\left(t_{90}\right)$, maximum $\left(M_{\max }\right)$ and minimum torque $\left(M_{\min }\right)$ and change in torque $\left(\Delta M=M_{\max }-M_{\min }\right)$ were obtained.

Next, elastomer compounds were placed in molds and heat using an electrical hydraulic press $\left(160{ }^{\circ} \mathrm{C}\right)$. The time of vulcanization was determined by rheometric measurements $\left(t_{90}\right)$.

The tensile test was performed using a tensile testing machine (Zwick, Ulm, Germany) equipped with an extensometer. The testing process was carried out at a crosshead speed of $500 \mathrm{~mm} / \mathrm{min}$ in accordance with standard ISO 37 by using standard dumbbell-shaped samples. The average value of tensile, elongation and modulus was recorded for five samples of composites.

The tear test was performed as per ISO 34 for three samples of each composite using the Zwick testing machine (Zwick, Ulm, Germany) with the test speed $50 \mathrm{~mm} / \mathrm{min}$. The specimen was cut to $100 \mathrm{~mm} \times 15 \mathrm{~mm} \times 1 \mathrm{~mm}$, "trousers" shape with a precut of $40 \mathrm{~mm}$ at the center.

The hardness was measured using a Shore A type durometer (Zwick, Ulm, Germany) and followed ISO 868 standard. The specimens were measured at 10 different locations on the composites. The average value of Shore A hardness number was calculated.

The cross-link densities $\left(v_{\mathrm{e}}\right)$ of straw-reinforced rubber composites were determined by an equilibrium swelling test, performed in toluene solvent at room temperature, based on the Flory-Rehner equation (Eqs. 1, 2) [22].

$$
v_{\mathrm{e}}=\frac{\ln \left(1-V_{\mathrm{r}}\right)+V_{\mathrm{r}}+\chi V_{\mathrm{r}}^{2}}{V_{0}\left(V_{\mathrm{r}}^{\frac{1}{3}}-\frac{V_{\mathrm{r}}}{2}\right)}
$$

where $v_{\mathrm{e}}$-the cross-link density $\left(\mathrm{mol} / \mathrm{cm}^{3}\right) ; V_{0}$ - the molecular volume of solvent $\left(106.7 \mathrm{~cm}^{3} / \mathrm{mol}\right) ; \chi$ - the Huggins parameter of the rubber-solvent interaction.

The Huggins parameters $(\chi)$ for rubber-toluene systems are given by the following equations [23-26]:

$$
\begin{aligned}
\mathrm{SBR}-\chi & =0.37+0.56 V_{\mathrm{r}} \\
\mathrm{EPM}-\chi & =0.43+0.34 V_{\mathrm{r}} \\
\mathrm{MVQ}-\chi & =0.45 \\
\mathrm{NBR}-\chi & =0.38+0.67 V_{\mathrm{r}}
\end{aligned}
$$

$V_{\mathrm{r}}$ is the volume fraction of elastomer in the swollen gel (Eq. 2).

$$
V_{\mathrm{r}}=\frac{1}{1+Q_{\mathrm{w}} \frac{\rho_{\mathrm{r}}}{\rho_{\mathrm{s}}}}
$$


where $Q_{\mathrm{w}}$-equilibrium swelling reduced by the filler content ( $\left.x[\mathrm{phr}]\right)$ $Q_{\mathrm{w}}=(100+x / 100) ; \rho_{\mathrm{r}}$-density of rubber $\left(\mathrm{g} / \mathrm{cm}^{3}\right) ; \rho_{\mathrm{s}}$ - density of solvent $\left(\mathrm{g} / \mathrm{cm}^{3}\right)$.

The damping properties of the vulcanizates were analyzed using a Zwick universal testing machine (Zwick, Ulm, Germany) in accordance with the PN-C-04289 standard. The specimens in this study were disk-shaped with diameter- $35 \mathrm{~mm}$ and height $-17.5 \mathrm{~mm}$. Each sample was stressed from 0 to $0.7 \mathrm{MPa}$, and then stress was reduced. Hysteresis loops were recorded, and the relative damping is the difference between deforming work and elastic recovery that value was determined according to Eq. 3:

$$
T_{\tau w}=\frac{\Delta W_{i}}{W_{\text {ibel }}} \cdot 100 \%
$$

where $T_{\tau w}$-relative damping, $\Delta W_{i}$-the difference between the compression work and the work during reducing the compressive stresses, $W_{\text {ibel }}$-compression work.

Dynamic mechanical analysis (DMA) was determined based on changes in the dynamic moduli as a function of the oscillation strain using a rotational rheometer (Ares G2 Rheometer, New Castle, USA) (plate-plate system, plate diameter: $20 \mathrm{~mm}$; gap $2 \mathrm{~mm}$ ). The test parameters are temperature: $25^{\circ} \mathrm{C}$, sample deformation rate: $10 \mathrm{rad} / \mathrm{s}$, stress: from 0.1 to $100 \%$, test force: $5 \mathrm{~N}$.

The simulation process of thermo-aging was carried out using a laboratory dryer (Binder, Tuttlingen, Germany) at the temperature of $70{ }^{\circ} \mathrm{C}$ for 14 days. To estimate the resistance of the samples to aging, their mechanical properties after aging were determined and compared with the values obtained for vulcanizates before the aging process. The aging factor $(K)$ was calculated as the numerical change in the mechanical properties of the samples upon aging (Eq. 4) [27]:

$$
K=\left(\mathrm{TS} \cdot E_{\mathrm{b}}\right)_{\text {after aging }} /\left(\mathrm{TS} \cdot E_{\mathrm{b}}\right)_{\text {before aging }}
$$

where TS is the tensile strength of the vulcanizate, and $E_{\mathrm{b}}$ is the elongation at break.

The morphology of the composite and straw was evaluated by means of scanning electron microscopy (SEM) with field emission (Hitachi, TM-1000, Tokio Japan). Before the SEM measurements, the samples were placed on carbon plasters and coated with the use of carbon target by the Cressington 208 HR system.

\section{Results and discussion}

\section{Rheometric properties}

Elastomers, in particular, those which do not contain double bonds, such as ethylene propylene rubber (EPM) or methyl-vinyl silicone rubber (MVQ), are crosslinked with organic peroxides. Peroxides are substances that vulcanize rubbers containing both unsaturated and saturated bonds due to the formation of $\mathrm{C}-\mathrm{C}$ transverse bonds via radical reactions. Vulcanization of rubber with elemental sulfur, which is the most common vulcanizing agent used for cross-linking of 
unsaturated rubbers [i.e., natural rubber (NR), styrene-butadiene rubber (SBR) or nitrile-butadiene rubber (NBR)], was discovered by C. Goodyear and by $\mathrm{T}$. Hancock over 170 years ago and found the widespread industrial implementation [28]. The application of sulfur curing systems leads to the forming of sulfuric bridges with a various number of sulfur atoms (mono-sulfuric $\mathrm{C}-\mathrm{S}-\mathrm{C}$, di-sulfuric $\mathrm{C}-\mathrm{S}_{2}-\mathrm{C}$ and poly-sulfuric cross-links.

$\mathrm{C}-\mathrm{S}_{x}-\mathrm{C}$, where $x$ can vary from 3 to 6 between elastomer chains [29]. For this reason, rubber mixtures are characterized by a different course of curves and rheometry parameters.

The effect of the type of rubber used and the content of bio-filler on the rheometry properties of the tested mixtures is shown in Fig. 1.

The scorch time $\left(t_{\mathrm{s} 2}\right)$ or time to initiate cure was determined from the increase in the minimum torque $\left(M_{\min }\right)$ value by $2 \%$, while the optimum cure time $\left(t_{90}\right)$ was determined from the time at the torque which reached to $90 \%$ of the maximum torque $\left(M_{\max }\right)$.

The cure time was found to be independent of straw loading but the unfilled rubber mixtures exhibited higher $t_{90}$ values. The difference of curing time (especially visible for SBR and NBR rubbers) was caused by the marching type of torque and the fact that the reference samples exhibited the lowest torque increase. In such circumstances, statistically the $90 \%$ torque increase has been reached later for the samples of lower total torque increase.

Moreover, the amount of heat generated in the mixing process is higher, the higher the viscosity. On the other hand, the indirect measure of the viscosity of the elastomer mixtures tested is the minimum torque values. For all types of rubber used, the viscosity increased with increasing the degree of filling of rubber blends, which also influenced the obtained vulcanization parameters. Based on the obtained results, it was found that the introduction of the filler into the rubber matrix (regardless of its type) resulted in the increase in the maximum torque $\left(M_{\max }\right)$ of the mixtures relative to the reference system. At the same time, this value increased along with the increase in filler content in the blends, which in turn was associated with an increase in their stiffness (stiffness). Filler particles reduce the mobility of the elastomer chain resulting in more rigid vulcanizates. Furthermore, with the increase in the fill level (regardless of the type of rubber used), an increase in the torque gain $\Delta M$ of the mixtures was also observed. The rising difference between the maximum and the minimum torque was probably the result of the increase in the hydrodynamic effect, the formation of a more complex "structure" of the filler and the increase in the cross-linking density of the vulcanizates (Table 2 ).

The main factor of the increase in the maximum torque value with an increase in the amount of filler was associated with the increase in the cross-linking density. The peroxide admixed in this form is extremely difficult to properly disperse and distribute due to high crystalline character and low chemical miscibility with rubbers. Therefore, in technological practice, DCP is applied on calcium carbonate carriers. The straw filler played the role of such carrier improving the dispersion and distribution of the peroxide, which resulted in higher cross-linking density. Similar effect could occur for the sulfur-based curing systems. 

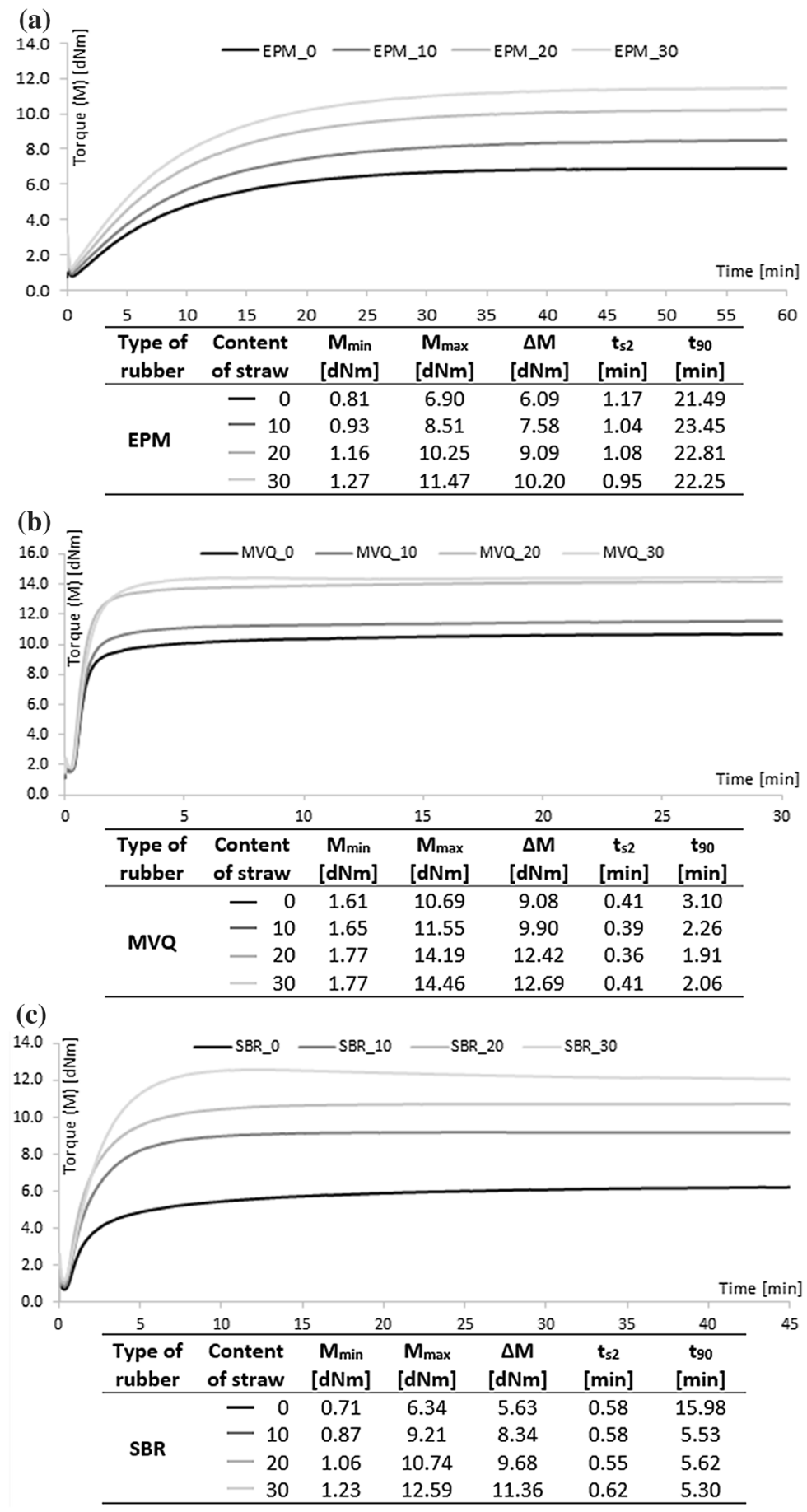

Fig. 1 Curing characteristics of a EPM rubber compounds, b MVQ rubber compounds, $\mathbf{c}$ SBR rubber compounds, $\mathbf{d}$ NBR rubber compounds 


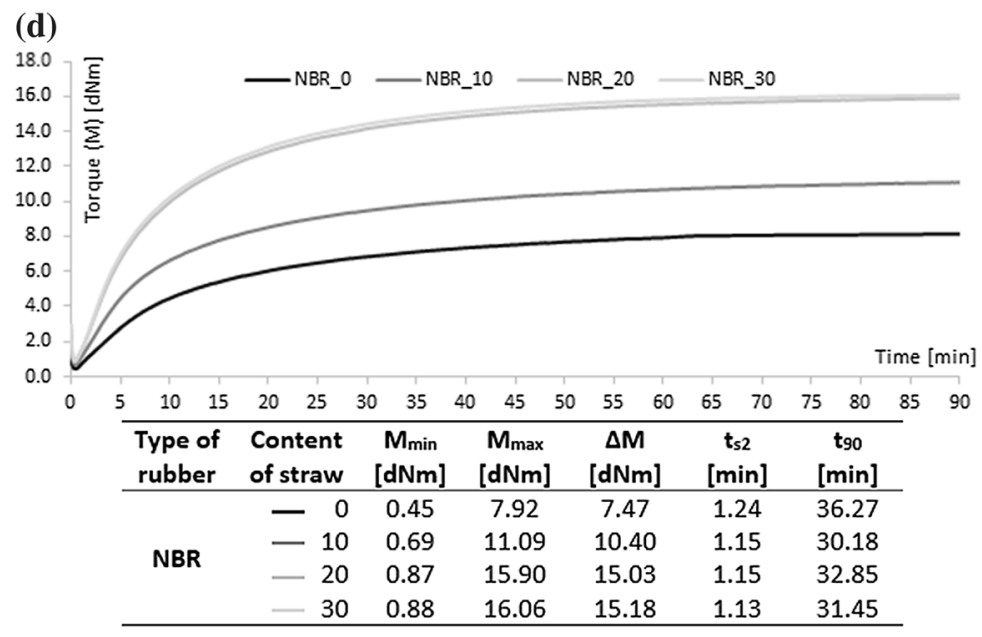

Fig. 1 (continued)

Table 2 The cross-linking density value of vulcanizates

\begin{tabular}{|c|c|c|c|c|}
\hline \multirow[t]{3}{*}{ Type of rubber } & \multicolumn{4}{|c|}{ Content of straw } \\
\hline & 0 & 10 & 20 & 30 \\
\hline & \multicolumn{4}{|c|}{$\nu_{\mathrm{e}} \times 10^{-5}\left(\mathrm{~mol} / \mathrm{cm}^{3}\right)$} \\
\hline EPM & 4.45 & $5.35(20.2 \%)$ & $6.43(44.5 \%)$ & $7.07(58.9 \%)$ \\
\hline MVQ & 18.10 & $25.36(40.1 \%)$ & $27.58(52.4 \%)$ & $30.46(68.0 \%)$ \\
\hline SBR & 1.42 & $1.44(1.4 \%)$ & $1.63(14.8 \%)$ & $1.65(16.2 \%)$ \\
\hline NBR & 5.22 & $5.95(14.5 \%)$ & $6.17(18.2 \%)$ & $6.45(23.6 \%)$ \\
\hline
\end{tabular}

$\nu_{\mathrm{e}}$-Cross-linking density value $\left(\mathrm{mol} / \mathrm{cm}^{3}\right)$

\section{Cross-linking density}

The application of sulfur systems leads to the forming of sulfuric cross-links between elastomer chains, while carbon-carbon bonds are formed in peroxide curing. One of the factors affecting the cross-linking process is the reaction of the rubber with the cross-linker. The created spatial structure depends on the type of rubber and the cross-linking agent used, which is confirmed by the data contained in Table 2. Other factors include chemical bonding of the rubber to the surface functional groups of the filler and physical interaction between the polymer matrix and the filler. The addition of a filler in the form of straw contributed to the increase in the value of the cross-linking density, which increased with the increasing amount of straw. The highest cross-linking density value was obtained for composites made of silicone rubber; moreover, the samples were characterized by the highest increases in cross-linking density in relation to the unfilled system. 
Comparing the data obtained for vulcanizates cured with a sulfur unit (NBR and SBR systems), the more developed spatial structure was exhibited by NBR composites. Also, the percentage increase in cross-linking density for these vulcanizates was greater than for SBR. One of the characteristic variables is the increase in $v_{\mathrm{e}}$ values for filled systems are the interactions between the elastomer and the filler. These connections act as physical network nodes, influencing the general characteristics of the spatial structure. Thus, the introduction of the filler into the polymer matrix affects the obtained values of the cross-linking density of the vulcanizates. The acrylonitrile-butadiene rubber contains in its structure polar acrylonitrile groups, which are more compatible with polar fibers. Hence, it is likely to achieve better adhesion at the interface between straw and elastomer.

Composites containing a sulfur cross-linking system showed slightly lower increases in the value of $v_{\mathrm{e}}$, but still significant compared to the unfilled reference sample (ca. 15-20\%). For the sulfur-cured samples, there is no possibility to form chemical bonds on rubber/filler interphase. Moreover, one of the factors contributing to the increase in network node concentration might have been the physical filler structure in the elastomer matrix. On the other hand, the dispersion improvement of the sulfuric system by the straw particles might have also been another important parameter. A more homogeneous distribution of cross-linking agents could have resulted in a more efficient cross-linking process, leading to higher $v_{\mathrm{e}}$ values of the overall system. Obtained significant increase (from 20 to 70\%) of the cross-linking density for the peroxide cured rubbers containing straw, could have indicated, what it was a possibility to generate filler/rubber via carbon-carbon bonds due to hydrocarbon structure of the filler.

\section{Dynamic mechanical analysis}

The above results were confirmed by dynamic mechanical analysis (Fig. 2).

The increase in the filler content resulted in an increase in the module value at low deformation amplitudes. The highest value of the $G^{\prime}$ module in the initial stage was exhibited by NBR straw composites. During the measurement, the reduction in the storage modulus as a function of deformation was noticeable. The decrease in the storage modulus with the increase in the deformation amplitude results from the disappearance of interactions - mainly van der Waals and hydrogen bonds of the newly formed secondary filler structure. This phenomenon is called the Payne effect. The filler-polymer interactions are related to the compatibility of the filler to the rubber and are the result of the rubber occlusion. In turn, the filler-filler interactions are the effect of mutual attraction of the filler particles and they testify to the ability to create a network, the so-called structure in the elastomeric medium. The highest decrease in the value of the storage modulus, so the strongest Payne Effect was also observed for composites made of acrylonitrile-butadiene rubber. It is clearly visible that for others than NBR rubbers when $30 \mathrm{phr}$ of the filler was added the beginning of the structure destruction started earlier than in case of the samples filled with 10 and 20 phr. This suggests that the dispersion and distribution of the filler in EPM, SBR and MVQ elastomers decreased with the increase in the filler amount. 

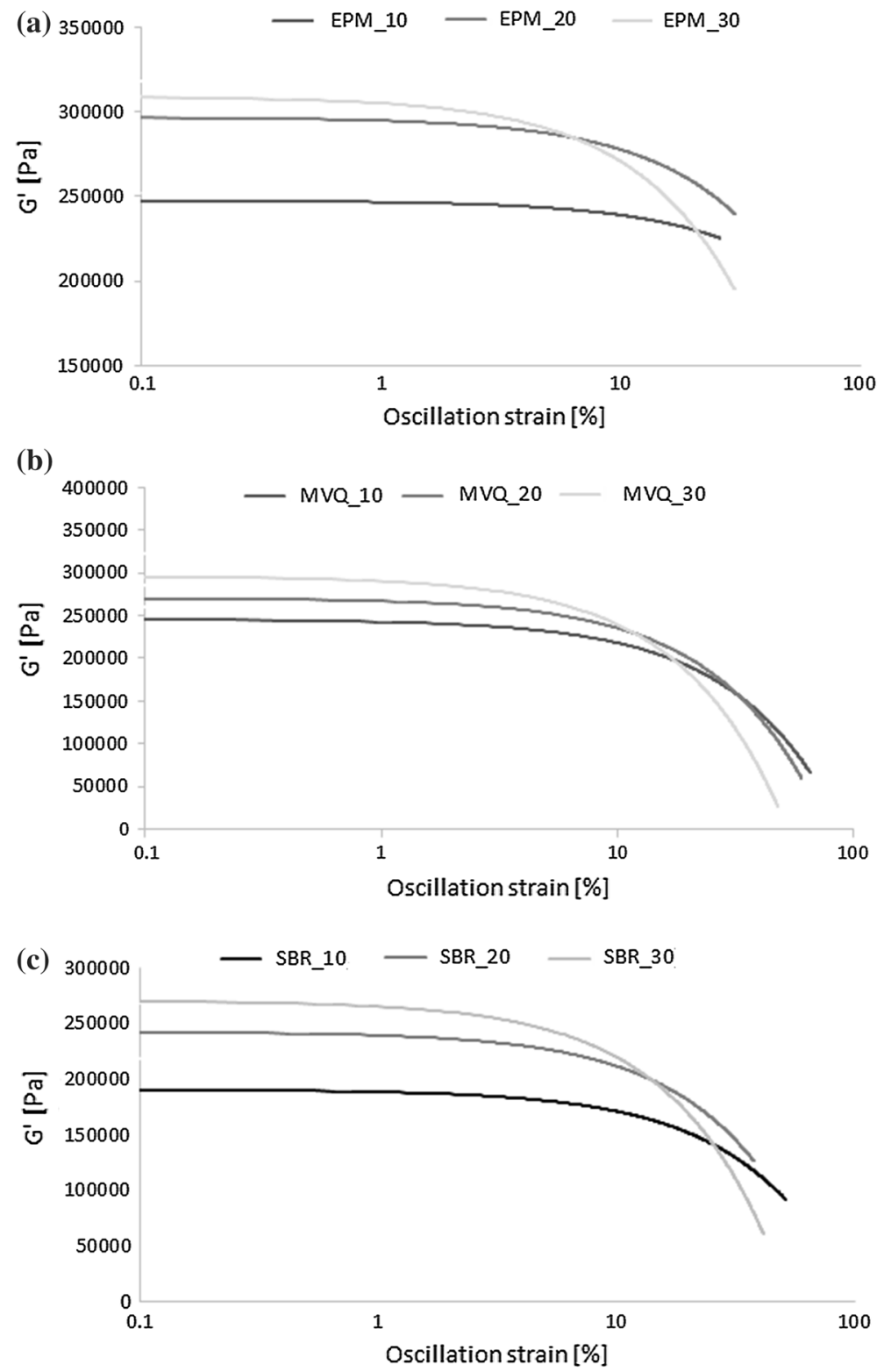

Fig. 2 Storage modulus $\left(G^{\prime}\right)$ versus oscillation strain for a EPM vulcanizates, b MVQ vulcanizates, c SBR vulcanizates, $\mathbf{d}$ NBR vulcanizates

However, for NBR, such tendency was not recorded suggesting that the dispersion for $30 \mathrm{phr}$ of the filler content was as good as for 20 and $10 \mathrm{phr}$. These results indicated the best compatibility of the NBR matrix with the filler used. That was influenced by the polarity of the medium (from the elastomers used) to the natural straw 


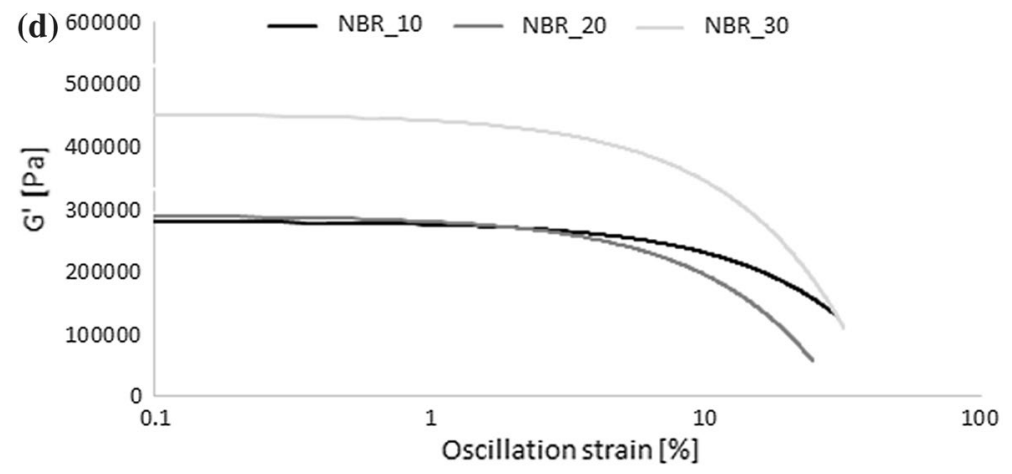

Fig. 2 (continued)

filler. SBR, MVQ, EPM, had a poor filler dispersion because they are considered as a non-polar rubber, respectively, compared with NBR whereas the hydroxyl groups on a straw surface provided a highly polar filler. Therefore, the hydroxyl groups could form hydrogen bonds resulting in a strong filler-filler interaction leading to poor filler dispersion. Compared to polar rubber filled with straw, NBR with its strong polar group produced a stronger filler-rubber interaction between the nitrile and hydroxyl groups, which normally give rise to an increase in the viscosity [30].

\section{Mechanical properties}

The cross-link density of the polymer composite and the filler structure produced in the elastomeric medium are the main factors affecting the mechanical behavior of the filled and reference vulcanizates. The effect of the incorporation of straw into elastomers on mechanical properties such as tensile strength, elongation at break (Fig. 3) hardness (Fig. 4), tear strength (Fig. 5) was studied.

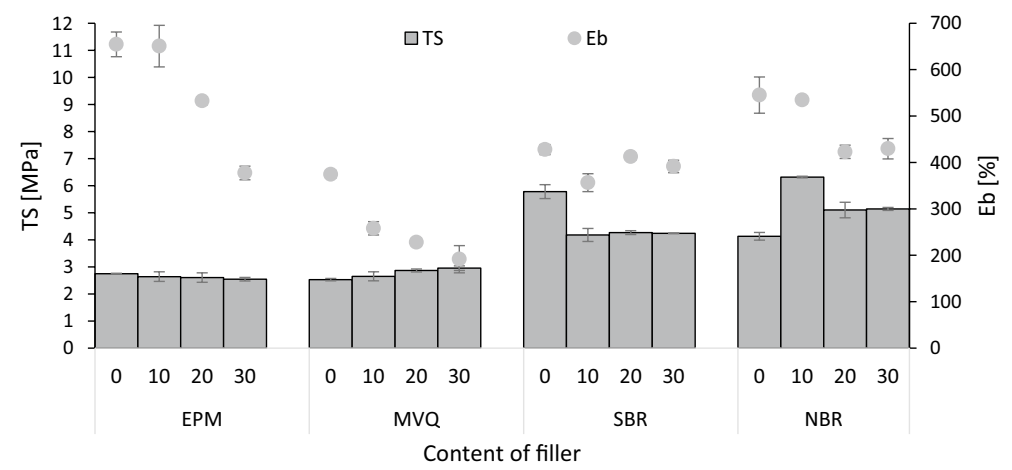

Fig. 3 Tensile strength (TS) and elongation at break $\left(E_{\mathrm{b}}\right)$ of vulcanizates 


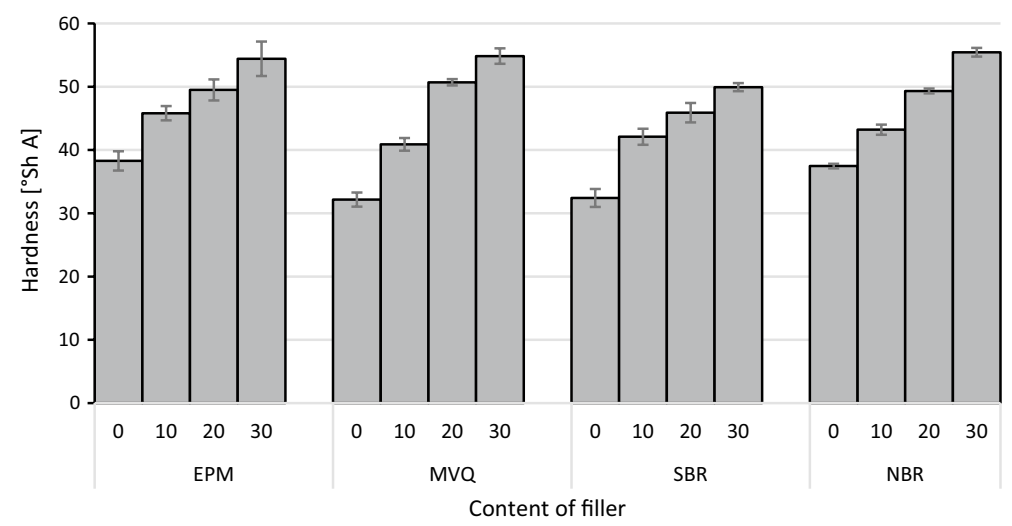

Fig. 4 Hardness of composites
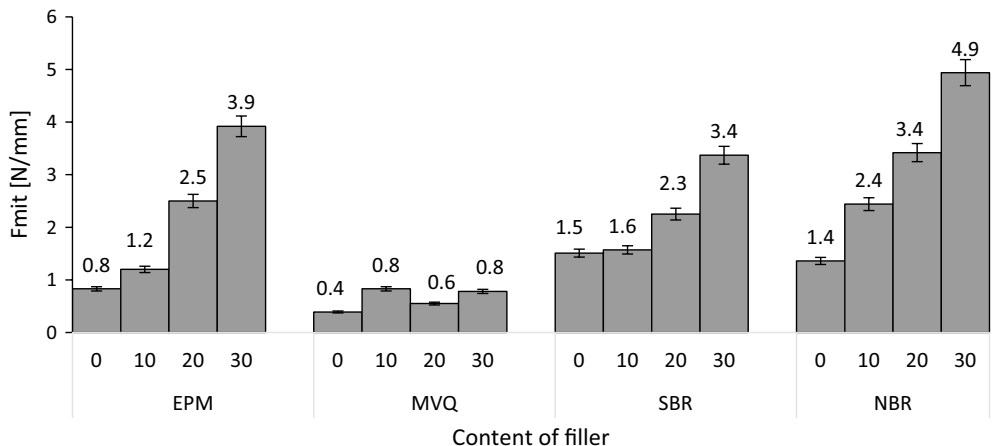

Content of filler

Fig. 5 Tear strength of composites ( $F_{\text {mit }}$ values - the average force needed to tear the sample)

The use of straw in a varied manner influenced the tensile strength properties. For the ethylene propylene and silicone elastomers, the addition of straw did not cause negative changes in tensile strength, regardless of the degree of filling. The hardness of reinforced elastomers depends on filler concentration and distribution. The hardness of the elastomeric composites increased for fiber composition of 10, 20 and 30 phr. The incorporation of straw enhanced the composites, making them harder and stiffer. The increases in filler volume fraction improved the modulus and hardness due to the enhancement of the cross-linking density. Straw composites, regardless of the polymer medium used, showed an increase in tear strength. With the higher the degree of filling, the stronger reinforcing effect was observed. It is due to the application of straw powder into elastomers. The part of short fibers being aligned along the direction of loading, which was perpendicular to the direction of tear propagation. Therefore, the short fibers transferred stress around and prevented crack growth. The concentration of short fibers increases 
tear strength by obstructing the tear path. The distribution of straw fibers can be seen in the SEM picture for a vulcanizate filled with 10 parts by weight (Fig. 6).

As the filler content increased, the highest $F_{\text {mit }}$ values (the average force needed to tear the sample) and their increments were obtained for composites prepared from an acrylonitrile-butadiene rubber. The higher polarity of the matrix promoted improved polymer-filler interfacial adhesion, resulting in increased tear strength.

\section{Thermo-oxidative aging}

Over time, the physical properties of the elastomeric products undergo various changes, as a result of which these products may become unusable due to excessive hardening, softening, cracking or other surface damage. Among the factors affecting the deterioration of elastomer properties, oxygen and elevated temperature are of the greatest importance. In order to check the resistance of elastomeric composites to aging materials, were subjected to thermo-oxidative aging simulation. Then, mechanical tests were carried out to determine changes in their performance. Based on the analysis of data contained in Figs. 7 and 8, the $K$ aging factor, which is an indicator of differences after degradation processes, showed varied values.

Physical properties depend on the structure of the cross-linked elastomer, related to the chemical structure of the rubber and the composite network. TS values of composites made of EPM and MVQ rubbers did not significantly change the strength parameters after aging compared to the results obtained for non-aged samples; hence, the value of $K$ coefficient was close to unity. A significant decrease in tensile strength after aging was observed for NBR and SBR composites. These are polymers containing in their structure unsaturated bonds, being elements particularly exposed to undesired oxidation reactions that negatively affect the characteristics of materials. Moreover, the elevated temperature initiates further cross-linking reactions of the elastomer, which contributes to the increase in the cross-linking density. The manifestation of the greater total number of nodes in the composites after simulated aging processes was the decrease in elongation at break values (Fig. 8). The filled composites, regardless of the matrix used, showed an increase in
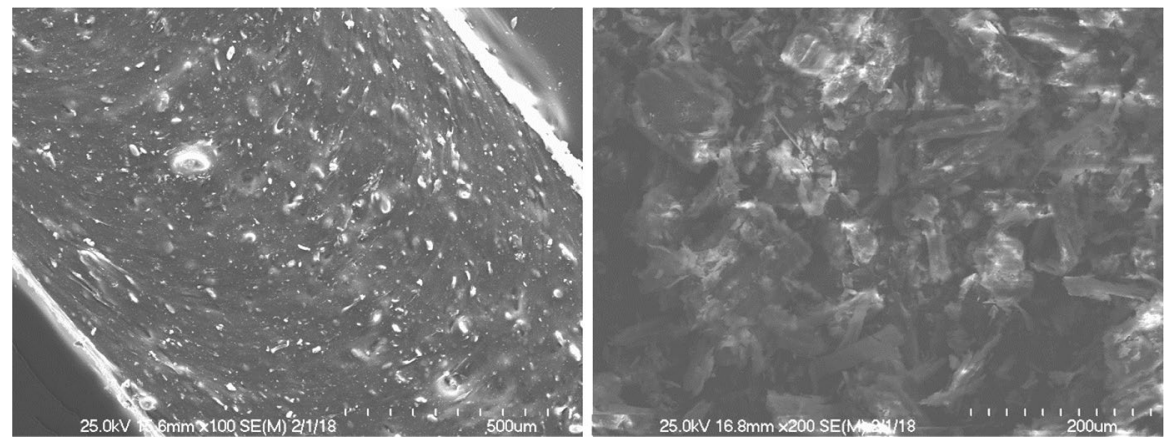

Fig. 6 SEM images of NBR composites filled with 10 phr straw (left) and cereal straw (right) 


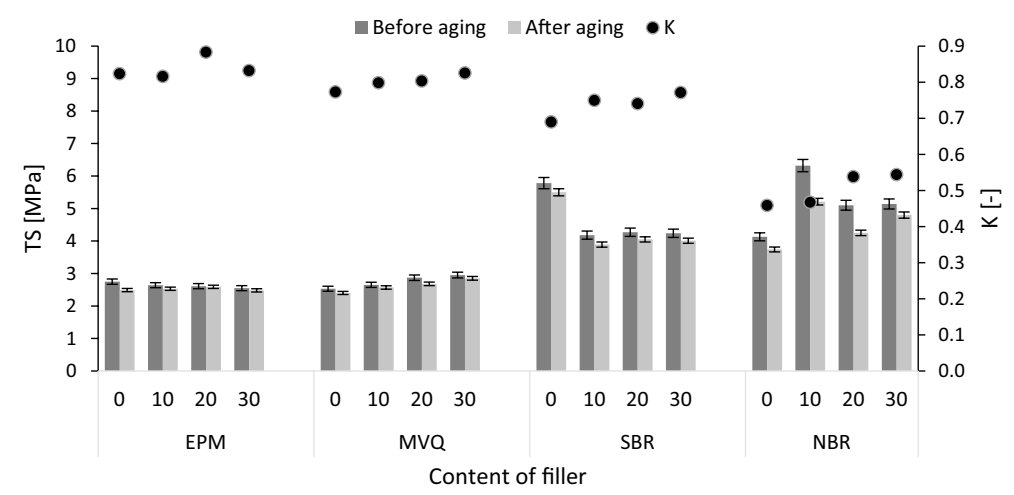

Fig. 7 Tensile strength of vulcanizates before and after aging or thermo-oxidative aging factors

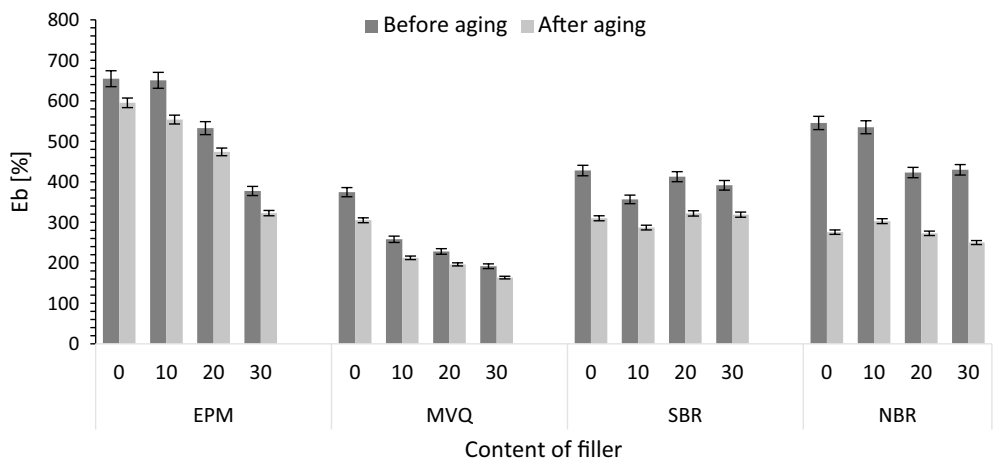

Fig. 8 Elongation at break of SBR vulcanizates before and after aging

resistance to thermo-oxidizing aging compared to the reference systems, what may be the result of antioxidant properties of lignin contained in cereal straw [31].

\section{Damping properties}

A characteristic feature of elastomeric materials is their high deformability in the direction of the applied stress. These materials have the ability to absorb (accumulate) a significant part of the energy used for their deformation and to partially disperse it in the form of heat. The ratio of the amount of energy dispersed to the amount of energy used to deform the sample allows determining the damping properties of the material, i.e., the ability to damp/diffuse vibrations. This parameter allows, among others to assess the long-term use of the material under cyclic loading conditions. The damping properties of the tested composites were determined on the basis of the calculated percentage values of the relative attenuation coefficient $T_{\tau w}$. The obtained results are presented graphically in Fig. 9. 


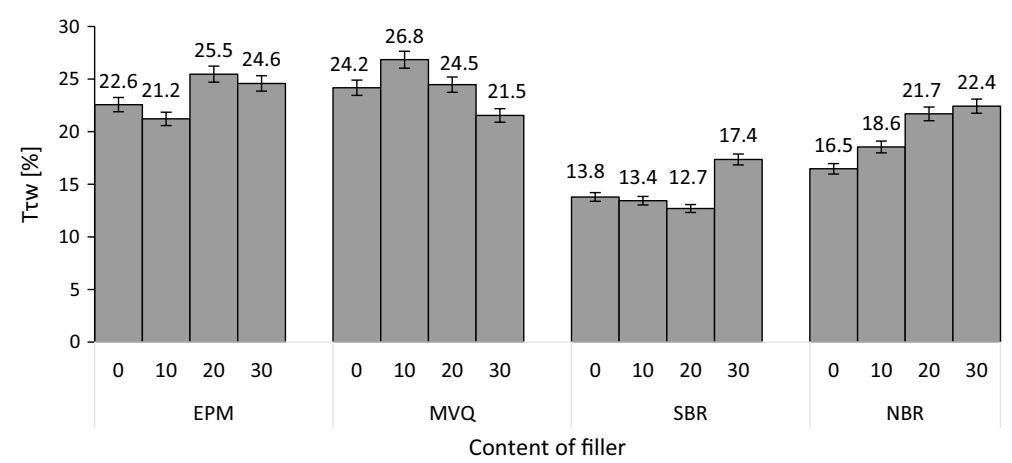

Fig. 9 Damping properties of rubber composites

Data analysis shows that the addition of lignocellulosic filler did not significantly affect the result of the obtained values of relative damping. For composites made of EPM, MVQ and SBR, no relationship was observed between the filler content and $T_{t w}$ values. In addition, the results obtained for filled vulcanizates did not differ significantly from the values obtained for reference systems. In the case of NBR samples, there was an increase in the relative damping coefficient as the content of straw in the elastomer increased. Furthermore, peroxide cross-linked composites (EPM, MVQ) showed higher values of relative attenuation coefficient compared to butadiene vulcanizates (NBR, SBR) cross-linked with a sulfuric vulcanized system.

\section{Conclusions}

The use of cereal straw, as a valuable additive to elastomer composites, creates opportunities for a new method of managing this material. The current methods of using production surpluses on cereal crops are insufficient or interfering with natural ecosystems, which further underline the importance of the research carried out. The ecological aspect is undoubtedly one of the important factors motivating potential implementations of the results obtained. The material analysis of newly created materials is also significant. The properties of biocomposites filled with ground cereal straw are characterized by improved functional properties. The wide range of rubber used highlights the application possibilities of straw. In addition, the appropriate selection of the elastomer matrix allows for the design of future composites using the potential of different polymeric centers specific properties.

The addition of straw influenced the cross-linking characteristics of the composites and shortened the vulcanization time. The use of this valuable lignocellulosic material as a rubber filler is also economically advantageous. Its additive has increased the volume of the products obtained while maintaining performance comparable with unfilled vulcanizates or as in the NBR/straw system improved tensile and tear strength of finished materials. Moreover, biocomposites produced on the basis of ethylene propylene and silicone rubber, gave the opportunity of long-term 
use of products, due to the high anti-aging resistance. Finally, these materials have shown high values of damping coefficients, which creates the basis for the production of deformation energy dissipative materials.

\section{Compliance with ethical standards}

Conflict of interest The authors declare that they have no conflict of interest.

Open Access This article is distributed under the terms of the Creative Commons Attribution 4.0 International License (http://creativecommons.org/licenses/by/4.0/), which permits unrestricted use, distribution, and reproduction in any medium, provided you give appropriate credit to the original author(s) and the source, provide a link to the Creative Commons license, and indicate if changes were made.

\section{References}

1. Sathishkumar TP, Navaneethakrishnan P, Shankar S, Rajasekar R (2014) Mechanical properties and water absorption of short snake grass fiber reinforced isophthallic polyester composites. Fibers Polym 15:1927-1934. https://doi.org/10.1007/s12221-014-1927-8

2. Sanjay M, Yogesha B (2017) Studies on natural/glass fiber reinforced polymer hybrid composites: an evolution. In: Materials today: proceedings, pp 2739-2747

3. Yusriah L, Sapuan SM, Zainudin ES, Mariatti M (2014) Characterization of physical, mechanical, thermal and morphological properties of agro-waste betel nut (Areca catechu) husk fibre. J Clean Prod 72:174-180. https://doi.org/10.1016/j.jclepro.2014.02.025

4. Dhakal HN, Sarasini F, Santulli C et al (2015) Effect of basalt fibre hybridisation on post-impact mechanical behaviour of hemp fibre reinforced composites. Compos Part A Appl Sci Manuf 75:5467. https://doi.org/10.1016/j.compositesa.2015.04.020

5. Vijaya Ramnath B, Manickavasagam VM, Elanchezhian C et al (2014) Determination of mechanical properties of intra-layer abaca-jute-glass fiber reinforced composite. Mater Des 60:643-652. https:// doi.org/10.1016/j.matdes.2014.03.061

6. Sanjay MR, Arpitha GR, Naik LL et al (2016) Applications of natural fibers and its composites: an overview. Nat Resour 7:108-114. https://doi.org/10.4236/nr.2016.73011

7. Deng Y, Paraskevas D, Tian Y et al (2016) Life cycle assessment of flax-fibre reinforced epoxidized linseed oil composite with a flame retardant for electronic applications. J Clean Prod 133:427-438. https://doi.org/10.1016/j.jclepro.2016.05.172

8. Al-Oqla FM, Sapuan SM (2014) Natural fiber reinforced polymer composites in industrial applications: feasibility of date palm fibers for sustainable automotive industry. J Clean Prod 66:347-354. https://doi.org/10.1016/j.jclepro.2013.10.050

9. $\mathrm{Ku} \mathrm{H}$, Wang H, Pattarachaiyakoop N, Trada M (2011) A review on the tensile properties of natural fiber reinforced polymer composites. Compos Part B Eng 42:856-873. https://doi.org/10.1016/j. compositesb.2011.01.010

10. La Mantia FP, Morreale M (2011) Green composites: a brief review. Compos Part A 42:579-588. https://doi.org/10.1016/j.compositesa.2011.01.017

11. Shubhra QTH, Alam AKMM, Gafur MA et al (2010) Characterization of plant and animal based natural fibers reinforced polypropylene composites and their comparative study. Fibers Polym 11:725-731. https://doi.org/10.1007/s12221-010-0725-1

12. Zakikhani P, Zahari R, Sultan MTH, Majid DL (2014) Extraction and preparation of bamboo fibrereinforced composites. Mater Des 63:820-828. https://doi.org/10.1016/j.matdes.2014.06.058

13. Lau K, Hung P, Zhu MH, Hui D (2018) Properties of natural fibre composites for structural engineering applications. Compos Part B Eng 136:222-233. https://doi.org/10.1016/j.composites b.2017.10.038 
14. John S, Nilmini PJD, Amandeep V, Wayne H (2010) A review of bast fibers and their composites. Part 1-fibers as reinforcements. Compos Part A 41:1329-1335. https://doi.org/10.1016/j.compo sitesa.2010.06.001

15. Pickering KL, Efendy MGA, Le TM (2016) A review of recent developments in natural fibre composites and their mechanical performance. Compos. Part A Appl. Sci. Manuf. 83:98-112

16. Faruk O, Bledzki AK, Fink HP, Sain M (2014) Progress report on natural fiber reinforced composites. Macromol Mater Eng 299:9-26

17. Masłowski M, Miedzianowska J, Strzelec K (2018) Silanized cereal straw as a novel, functional filler of natural rubber biocomposites. Cellulose. https://doi.org/10.1007/s10570-018-2093-8

18. Masłowski M, Miedzianowska J, Strzelec K (2018) Influence of peroxide modifications on the properties of cereal straw and natural rubber composites. Cellulose 25:4711-4728. https://doi. org/10.1007/s10570-018-1880-6

19. Masłowski M, Miedzianowska J, Strzelec K (2017) Natural rubber biocomposites containing corn, barley and wheat straw. Polym Test 63:84-91. https://doi.org/10.1016/j.polymertesting.2017.08.003

20. Panthapulakkal S, Sain M (2015) The use of wheat straw fibres as reinforcements in composites. In: Faruk O, Sain M (eds) Biofiber reinforcements in composite materials. Woodhead Publishing, Toronto, pp 525-549

21. Palmieri N, Forleo MB, Giannoccaro G, Suardi A (2017) Environmental impact of cereal straw management: an on-farm assessment. J Clean Prod 142:2950-2964. https://doi.org/10.1016/j.jclep ro.2016.10.173

22. Flory PJ, Rehner J (1943) Statistical Mechanics of Cross-Linked Polymer Networks I. Rubberlike Elasticity. J Chem Phys 11:512-520. https://doi.org/10.1063/1.1723791

23. Maciejewska M, Zaborski M (2018) Ionic liquids as coagents for sulfur vulcanization of butadienestyrene elastomer filled with carbon black. Polym Bull 75:4499-4514. https://doi.org/10.1007/s0028 9-018-2281-6

24. Lipińska M, Zaborski M (2017) Influence of POSS addition on properties of ethylene-propylene EPM and hydrogenated butadiene-acrylonitrile HNBR composites containing layered silicate and nanometric silica. Int Res J Adv Eng Sci 2:2455-9024

25. Frounchi M, Dadbin S, Panahinia F (2006) Comparison between electron-beam and chemical crosslinking of silicone rubber. Nucl Instrum Methods Phys Res Sect B Beam Interact Mater Atoms 243:354-358. https://doi.org/10.1016/j.nimb.2005.09.013

26. Maciejewska M, Zaborski M (2014) Effect of ionic liquids on the dispersion of zinc oxide and silica nanoparticles, vulcanisation behaviour and properties of NBR composites. Express Polym Lett 8:932-940. https://doi.org/10.3144/expresspolymlett.2014.94

27. Masek A, Zaborski M, Kosmalska A, Chrzescijanska E (2012) Eco-friendly elastomeric composites containing Sencha and Gun Powder green tea extracts. C R Chim 15:331-335. https://doi. org/10.1016/j.crci.2011.11.013

28. Shahrampour H, Motavalizadehkakhky A (2017) The effects of sulfur curing systems (insolublerhombic) on physical and thermal properties of the matrix polymeric of styrene butadiene rubber. Pet Chem 57:700-704. https://doi.org/10.1134/S0965544117080138

29. Kruzelák J, Dosoudil R, Sýkora R, Hudec I (2017) Rubber composites cured with sulphur and peroxide and incorporated with strontium ferrite. Bull Mater Sci 40:223-231. https://doi.org/10.1007/ s12034-016-1347-z

30. Arayapranee W, Rempel GL (2013) Effects of polarity on the filler-rubber interaction and properties of silica filled grafted natural rubber composites. J Polym 2013:1-9. https://doi. org/10.1155/2013/279529

31. Li Z, Ge Y (2012) Antioxidant activities of lignin extracted from sugarcane bagasse via different chemical procedures. Int J Biol Macromol 51:1116-1120. https://doi.org/10.1016/j.ijbio mac.2012.09.004

Publisher's Note Springer Nature remains neutral with regard to jurisdictional claims in published maps and institutional affiliations. 